Volume 8

Issue 2

2011
$\mathrm{M}$ indfulness can be defined as paying attention to one's inner and outer experiences in a non-judgmental manner from moment to moment. (Kabat-Zinn, 2003) When we are mindful we are more aware of the current moment and we simply observe our thoughts, feelings and sensations as they are without reacting to or trying to change them.

Most of the time we are immersed in a constant stream of inner experience without being aware of it, or of how our thoughts, feelings, desires and impulses influence us. Frequently our thoughts, whether about illness, not getting what we want, problems from the past or worries about the future, make us feel angry, anxious or depressed. By cultivating mindfulness we can learn to step back from our inner and outer experience, freeing us from habitual patterns of reacting to it or judging it. Instead, we can learn to live in the present moment and to make wise choices about how we respond to the mental and physical events in our lives.

Although mindfulness practice may result in relaxation and a calm mind, mindfulness is a skill that can be applied to be present with any state, including arousal, habitual reactions, and physical or emotional pain. Mindfulness has been practiced for thousands of years in different spiritual traditions. In 1979, Dr. Jon Kabat-Zinn developed a mindfulness-based curriculum at UMass Medical Center to help medical patients incorporate mindfulness practice in their daily life to help them cope with stress, chronic pain and other chronic

\section{Mindfulness and Health} Carl Fulwiler and Fernando de Torrijos

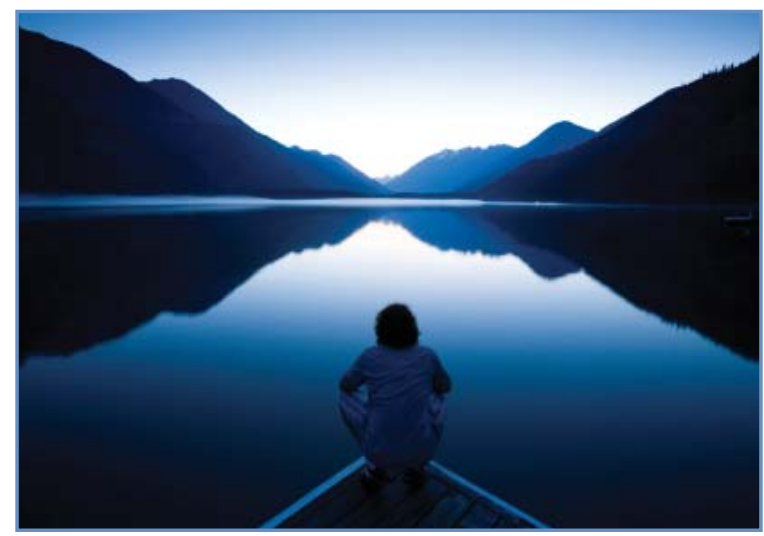

medical conditions. (Kabat-Zinn, 1990) This program, called Mindfulness-Based Stress Reduction (MBSR), has been replicated around the world and helped spark the growing popularity of mindfulness practices. (Ludwig, 2008)

Adaptations of MBSR followed, including:

- Mindfulness-Based Cognitive Therapy (MBCT) for prevention of recurrence of depression (Segal et al., 2002)

- Mindfulness-Based Relapse Prevention (MBRP) for prevention of substance abuse relapse (Chawla et al., 2010)

- Mindfulness has also been incorporated into other cognitivebehavioral interventions such as Dialectical Behavior Therapy (DBT; Linehan, 1993) and Acceptance and Commitment Therapy (ACT; Hayes et al., 2004)

\section{Research on Mindfulness}

Mindfulness-based interventions have been found to be beneficial for a number of psychological and physical conditions such as chronic pain, depression, anxiety, addictions 
Mindfulness is developed through a variety of formal meditation practices, and informal practices applied in daily life.

Formal mindfulness practices include:

- $\quad$ sitting meditation

- body scan

- mindful yoga

- walking meditation

- loving-kindness meditation

Informal practices bring full awareness to everyday activities such as eating, breathing, walking, washing the dishes, brushing our teeth, etc.

and personality disorder. (Grossman, 2004) It has been shown that participating in an 8-week MBSR training produces changes in the brain function and the immune system conducive to wellness. Research has shown that mindfulness practice leads to improvements in brain functions that help us to respond better to stressful and upsetting conditions and situations, like chronic pain, or upsetting thoughts.

At the Center for Mental Health Services Research at UMass, the authors are conducting research on how mindfulness affects physical and emotional well-being. In one project we are developing a mindfulness-based intervention for people with lived experience of trauma and substance abuse in prison. In another we are collaborating with other researchers at UMass to study changes in brain circuitry involved in the beneficial effects of mindfulness. Finally, we are studying the benefits of mindfulness trainings conducted by $\mathrm{Mr}$. de Torrijos with medical students, residents, inpatient staff, and inpatients in the UMass Dept. of Psychiatry and at Worcester State Hospital.

\section{Recommendations for a Mindfulness-Based Approach to Wellness}

The stress response is our body's way of dealing with any threat, pressure or demand. In true emergencies it activates our fight or flight mechanism of selfprotection. But most of the time the stress response is triggered by our automatic, or habitual, reactions to situations. Chronic or repeated activation of the stress response leads to health problems and reduces our quality of life. Mindfulness works to reduce stress and increase our ability to enjoy life by bringing awareness to these automatic reactions and, in doing so, breaking the chain of habitual responses.

Mindfulness-based stress reduction can be part of a holistic approach that encourages people to take responsibility for improving their health and wellbeing. Learning to pay attention and be present with whatever arises in experience encourages the development of health-promoting behavior changes, including informed exercise, nutritional awareness, and improved sleep. In addition, it promotes changes in unhealthy behaviors such as smoking, emotional eating, and alcohol and drug use.

Mindfulness offers a foundation for creating a "culture of wellness" for all stakeholders involved in the personcentered approach to healthcare. Acknowledgement of the holistic nature of individuals is fundamental to mindfulness practices which focus on the whole person, including the physical, mental and spiritual dimensions. Mindfulness is also strength-based and focused on what a person can choose to do to cultivate their own well-being using the wisdom and vast potential of inner resources available to everyone. Many employers and healthcare organizations are offering mindfulness training to staff and patients because it confers benefits such as improved stress management and well-being, enhanced spirituality, increased capacity for empathy, and reductions in anxiety and burn-out.

In randomized studies, MBSR participants have shown significant brain and immune changes compared with control groups:

- A study conducted in a workplace setting found that MBSR participants demonstrated a shift in left-sided EEG activation over prefrontal cortex, an area associated with positive affect, and significantly greater immune response following influenza vaccination compared to controls. (Davidson et al., 2003)

- A recent study of MBSR found increases in volume of the hippocampus, an area associated with learning and memory, and reductions in volume of the amygdala, a region associated with anxiety and stress. (Hölzel et al., 2011) Moreover, the decrease in amydala volume correlated with reductions in stress levels. 


\section{References}

Grossman, P., L. Niemann, et al. (2004). "Mindfulness-based stress reduction and health benefits. A metaanalysis." J Psychosom Res 57(1): 35-43.

Kabat-Zinn, J. (1990). "Full Catastropher Living: Using the Wisdom of Your Body and Mind to Face Stress." Pain and Illness, Delacorte, NY.

Kabat-Zinn, J. (2003). "Mindfulness-based interventions in context: past, present, and future." Clinical Psychology: Science \& Practice 10(2): 144-156.

Ludwig, D. S. and J. Kabat-Zinn (2008). “Mindfulness in medicine.” JAMA 300(11): 1350-1352.

Mindfulness and Health - C. Fulwiler \& F. de Torrijos 